\title{
Influence of Strong Magnetic Fields on Laser Pulse Propagation in Underdense Plasma
}

\author{
T C Wilson ${ }^{1}$, F Y Li ${ }^{1}$, M Weikum ${ }^{1,2}$, Z M Sheng ${ }^{1,3,4}$ \\ ${ }^{1}$ SUPA, Department of Physics, University of Strathclyde, Glasgow G4 0NG, \\ United Kingdom \\ 2 Deutsches Elektronensynchrotron (DESY), 22607 Hamburg, Germany \\ 3 Key Laboratory for Laser Plasmas (MoE) and Department of Physics and \\ Astronomy, Shanghai Jiao Tong University, Shanghai 200240, China \\ ${ }^{4}$ Collaborative Innovation Center of IFSA, Shanghai Jiao Tong University, \\ Shanghai 200240, China
}

Submitted to: Plasma Phys. Control. Fusion 
Abstract. We examine the interaction between intense laser pulses and strongly magnetised plasmas in the weakly relativistic regime. An expression for the electron Lorentz factor coupling both relativistic and cyclotron motion nonlinearities is derived for static magnetic fields along the laser propagation axis. This is applied to predict modifications to the refractive index, critical density, group velocity dispersion and power threshold for relativistic self-focusing. It is found that electron quiver response is enhanced under right circularly-polarised light, decreasing the power threshold for various instabilities, while a dampening effect occurs under left circularly-polarised light, increasing the power thresholds. Derived theoretical predictions are tested by one and three-dimensional particle-in-cell simulations.

\section{Introduction}

Static magnetic fields induce cyclotron motion in the charged particles of a plasma. The particles gyrate at a frequency proportional to the applied field, and this motion modifies the optical properties of the plasma. Classical plasma theory is also altered by the relativistic mass increase of electrons when the driving laser amplitude is high enough, and this couples to the magnetic nonlinearity through mass dependence of the cyclotron frequency. In this way, magnetic and relativistic effects are linked, and must be considered together.

This work focuses exclusively on the propagation of circularly polarised light along the direction of an externally applied DC magnetic field. In this case, there is distinct difference between right circular polarisation (RCP) and left circular polarisation (LCP) of the light, which are respectively with clockwise and anticlockwise rotation along the laser propagation direction. A dramatic effect of magnetisation on RCP light is greatly increased plasma transparency, which allows for novel schemes of efficient, magnetically guided plasma acceleration [1]. This is of particular relevance to the fast-ignition scheme of inertial confinement fusion, which relies heavily on precise deposition of energy by accelerated particles [2]. This motivates an understanding of the physics of intense laser interactions with highly magnetised plasma. For lasers on the order of $\mu \mathrm{m}$ wavelength, these conditions necessitate field strengths on the order of $10^{4}$ Tesla.

The generation of ultra-strong magnetic fields has received considerable interest in the last decade. Longlasting fields of around 100 Tesla can now be readily produced with conventional techniques [3, 4]. Higher strength fields operating at shorter timescales are also produced [5]. High power laser interaction with dense plasma can even produce quasi-static magnetic fields at the $10^{5} \mathrm{~T}$ level $[6,7,8,9]$. By irradiating a capacitor-coil target with kilo-Joule high power lasers, it is demonstrated experimentally that high magnetic fields at kT level can be produced $[10,11]$. The latter is particularly interesting for a number of applications such as inertial confinement fusion [12].

Even in the linear wave regime, the magnetic fields result in unique effects of wave propagation. Observations of anomalous radio waves were documented as early as 1894 , termed whistlers due to the descending tone heard as the waves were picked up on receivers. Explanations of the physical processes underlying them developed in the 1950s as general understanding of plasma physics advanced [13]. The comparatively high frequency of laser light compared to radio waves has, until relatively recently, precluded the investigation of whistler mode lasers. However, advances in laser technology and the creation of high strength magnetic fields now allow access to this parameter regime, and has spurred interest in the combination of magnetic and relativistic plasma effects.

Nonlinear propagation of lasers in unmagnetised plasma has been widely studied. The key physics involving the propagation of a laser pulse in a plasma concern the transverse evolution $[14,15,16]$, typically characterised by self-focusing (SF) processes, and the longitudinal evolution, governed by processes such as self-phase modulation (SPM), $[17,18]$ which may act to compress an already short pulse in plasma further. The vast majority of this work has already been undertaken. More recently work has been done determining the effect magnetisation plays on the propagation of lasers. The transverse evolution of lasers in weakly-relativistic magnetised plasmas is investigated by [19, 20], who conclude that magnetic field enhances self-focusing of $\mathrm{RCP}$ pulses. Longitudinal effects are investigated by some researchers [21, 22, 23], who conclude that SPM may be enhanced for RCP light, or inhibited for LCP light, by an applied magnetic field.

In this work, we consider the strongly magnetised regime, in which the electron cyclotron frequency is on the order of the incident laser frequency. In this case, magnetic fields dramatically influence the magnitude and rate of the SF and SPM processes with a much lower power threshold for RCP light. In section 2, a theory model is presented for a regime where the laser pulse duration is relatively long 
so that ponderomotive force and subsequent density perturbations can be neglected. Therefore we focus on the effect of strong magnetic fields on relativistic SF and relativistic SPM. Also the self-generated magnetic fields $[24,25,26,27,28]$ are neglected as compared with the externally applied magnetic field. Results from self-consistent three-dimensional particle-in-cell simulations are presented in section 3 , which confirm the theory predictions given in section 2. Simulations also illustrate complicated longitudinal and transverse couplings at later stage of the laser propagation. The paper concludes with a summary in section 4 .

\section{Theory Model}

\subsection{Dispersion Relation}

We aim to describe the characteristics of propagation of a laser beam in a magnetised underdense plasma, with the static magnetic field aligned along the propagation axis of the laser and with the cyclotron frequency comparable to the laser frequency. We may do this by generalisation of the treatment used to study Faraday rotation [29]. The equation of motion for a cold, magnetised, relativistic electron fluid is

$\frac{\partial}{\partial t}(\boldsymbol{v} \gamma)=-\frac{e}{m_{e}}\left[\boldsymbol{E}_{\boldsymbol{l}}+\boldsymbol{v} \times\left(\boldsymbol{B}_{\boldsymbol{l}}+\boldsymbol{B}_{\boldsymbol{s}}\right)\right]$,

where $m_{e}$ and $e$ are the electron rest mass and charge respectively, $\boldsymbol{E}_{\boldsymbol{l}}$ and $\boldsymbol{B}_{\boldsymbol{l}}$ correspond to the rapidly varying fields of the laser, $\boldsymbol{B}_{\boldsymbol{s}}$ is the static magnetic field along the laser axis, $\gamma=\left(1-v^{2} / c^{2}\right)^{-1 / 2}$ is the electron Lorentz factor, $\boldsymbol{v}$ is the electron velocity and $v=|\boldsymbol{v}|$. The electric field of a circularly polarised laser is given by

$\boldsymbol{E}_{\boldsymbol{l}}=\Re\left\{\hat{\boldsymbol{e}} E_{0} \exp [i(k z-\omega t)]\right\}$,

where $i(k z-\omega t)$ is the rapidly varying phase term, in which $\omega$ and $k$ are the vacuum frequency and wavenumber respectively, $E_{0}$ is the electric field amplitude and $\hat{\boldsymbol{e}}=\left(\hat{\boldsymbol{e}}_{\boldsymbol{x}}+i \delta \hat{\boldsymbol{e}}_{\boldsymbol{y}}\right)$ describes the polarisation of the laser, with $\hat{\boldsymbol{e}}_{\boldsymbol{x}}$ and $\hat{\boldsymbol{e}}_{\boldsymbol{y}}$ unit vectors in $x$ and $y$, respectively, and $\delta$ denoting the direction of rotation with $\delta=+1$ corresponding to RCP and $\delta=-1$ corresponding to LCP.

Analytically, we take the long-pulse approximation such that pulse duration $L \gg 2 \pi / \omega_{p}[16]$ and the pulse amplitude does not vary much over a laser period. Hence, plasma electron wave excitation and longitudinal ponderomotive force may be considered to be low. We hence take $\boldsymbol{v} \simeq \boldsymbol{v}_{\perp}$, given by

$\boldsymbol{v}_{\perp}=\Re\left\{-\frac{i \eta e E_{0}}{m_{e} \omega \gamma} \hat{\boldsymbol{e}} E_{0} \exp [i(k z-\omega t)]\right\}$.

Borrowing some convenient notation from [30], $\eta=$ $\left(1-\delta \omega_{c} / \gamma \omega\right)^{-1}$ and $\omega_{c}=e B_{s} / m_{e}$ is the electron cyclotron frequency. For further convenience, we may also define $B=\omega_{c} / \omega$. We now arrive at an implicit expression for the time-averaged electron Lorentz factor

$\gamma=\sqrt{1+\left(\frac{e E_{0} \eta}{m_{e} \omega c}\right)^{2}}$.

The inhomogeneous electromagnetic wave equation is

$\left(\nabla^{2}-\frac{1}{c^{2}} \frac{\partial^{2}}{\partial t^{2}}\right) \boldsymbol{E}_{\boldsymbol{l}}=-\mu_{0} \frac{\partial \boldsymbol{J}_{\perp}}{\partial t}$,

with the transverse current density $\boldsymbol{J}_{\perp}=-e n_{e} \boldsymbol{v}_{\perp}$. Here, $\mu_{0}$ is the magnetic permeability of free space and $n_{e}$ is the electron number density. Now introduce the normalisation $\boldsymbol{a}=e \boldsymbol{E}_{\boldsymbol{l}} / m_{e} \omega c$ for the field amplitude, and substitutions $\tilde{n}_{e}=n_{e} / n_{0}, \omega_{p}^{2}=e^{2} n_{0} / m_{e} \epsilon_{0}$ and $k_{p}=\omega_{p} / c$, with the tilde henceforth dropped for convenience. Here $\epsilon_{0}$ is the electrical permittivity of free space, $n_{0}$ is the background electron density and $\omega_{p}$ and $k_{p}$ are the plasma frequency and wavenumber, respectively. The corresponding wave equation in magnetised plasma can hence be found as

$$
\left(\nabla^{2}-\frac{1}{c^{2}} \frac{\partial^{2}}{\partial t^{2}}\right) \boldsymbol{a}=\frac{k_{p}^{2} n_{e} \eta}{\gamma} \boldsymbol{a}
$$

Under the assumption that the plasma is homogeneous, the density profile $n_{0}$ is constant. Consider a simple plane wave $a=\exp [i(k z-\omega t)]$. By substituting this and $n_{e}=1$. into (6), we can recover the dispersion relation for circularly polarised light with an applied magnetic field as

$\omega^{2}=k^{2} c^{2}+\omega_{p}^{2} \frac{\eta}{\gamma}$,

which is consistent with that given in previous work $[1,30]$, and illustrated in Figure 1 . It shows that when $\omega_{c} \neq 0$, cutoff frequencies for RCP and LCP light take different values. These may be obtained by setting $k=0$ in (7) and have the general form $\omega_{R, L}=$ $\left[\left(\omega_{c} / 2 \gamma\right)^{2}+\omega_{p}^{2} / \gamma\right]^{1 / 2} \pm \omega_{c} / 2 \gamma$. The cutoff frequency is reduced down to $\omega_{L}$ for LCP light, and increased up to $\omega_{R}$ for RCP light. While transparency is increased for LCP light, there is still a cutoff frequency below which no propagation is possible. Conversely, RCP waves may also propagate in whistler mode, creating an opaque frequency range $\omega_{c} / \gamma<\omega<\omega_{R}$.

From Eq. (7) one may immediately obtain the normalised phase velocity as $\beta_{p}=$ $\left(1-(\eta / \gamma)\left(\omega_{p} / \omega\right)^{2}\right)^{-1 / 2}$. The plasma refractive index $N$ and dielectric function $\epsilon$ are related by $\epsilon=N^{2}=$ $1-(\eta / \gamma)\left(\omega_{p} / \omega\right)^{2}$. We may also evaluate $\partial \omega / \partial k$ for the normalised group velocity

$\beta_{g}=N\left[1+\frac{n \eta^{2}}{2 \gamma^{2}}\left(\delta B-\frac{a^{2} \eta^{3}}{\gamma+\delta B a^{2} \eta^{3} \gamma^{-2}}\right)\right]^{-1}$,

where we have used $\gamma=\sqrt{1+a^{2} \eta^{2}}$ and $n=\left(\omega_{p} / \omega\right)^{2}$. $\mathrm{RCP}$ light may propagate in the whistler mode, where 


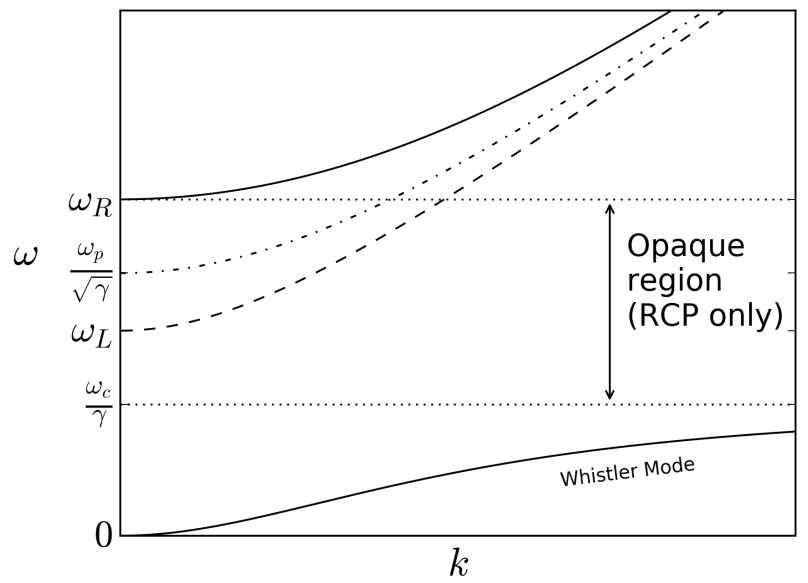

Figure 1: The magnetised plasma dispersion relation. The solid curve represents RCP light, the dashed curve represents LCP light and the dot-dashed curve indicates the unmagnetised dispersion for comparison.

$B / \gamma>1$ and hence $N>1$. Under these conditions, propagation is possible in overdense plasmas [31].

Extra care must be taken for intense light in the whistler mode. Due to relativistic electron mass increase, it is possible for the effective cyclotron frequency to be lowered enough that it approaches the laser frequency and the plasma is returned to a resonant state.

Consider the inverse of $(4) ; a=\left[\left(\gamma^{2}-1\right) / \eta^{2}\right]^{1 / 2}$. In whistler mode, this function reveals a critical point at $\partial a / \partial \gamma=0$ beyond which the plasma is brought to a near-resonant, strongly absorbing state. This may be expressed in terms of $B$ as a critical laser amplitude

$a_{c}=\left(B^{2 / 3}-1\right)^{3 / 2}$,

or a critical gamma $\gamma_{c}=B^{1 / 3}$. These quantities do not correspond directly to the resonant point of $B / \gamma=1$, rather they represent the point at which strong heating begins and large amounts of energy are transfered from the laser to the bulk plasma. As $B$ is increased, the critical point is increased in turn, as is shown in Figure 2.

The frequency gap associated with RCP light results in an opaque region close to the resonant point occupying the range $(\gamma-n)<B<\gamma$. In reality, this is a poor definition of the actual range at which a laser may still propagate in the plasma, as strong absorption occurs even outside this region. As a result, the insight gained from this is of little practical use. However, at the very least we can estimate the upper bound as $\gamma_{c}$. This improves our estimate to $(\gamma-n)<B<\gamma^{3}$. We show the effect of increasing $a$ on $\beta_{g}$ in Figure 3.

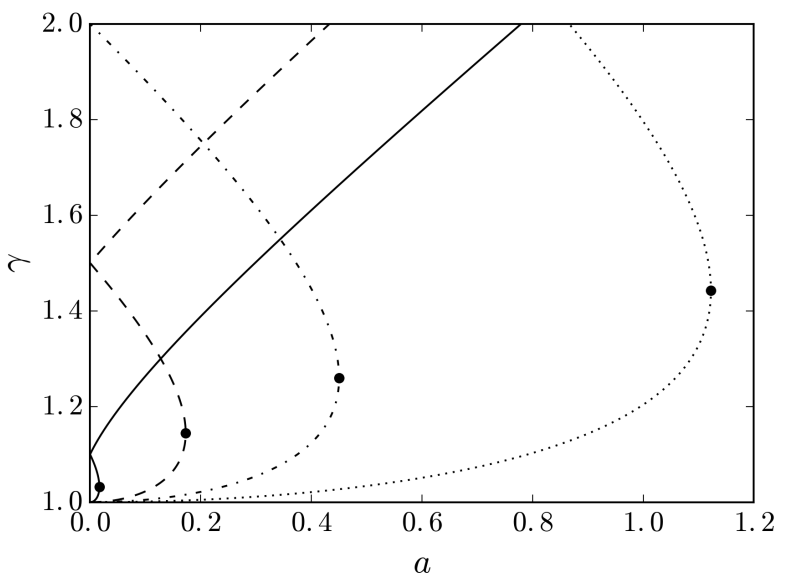

Figure 2: The calculated relationship between $\gamma$ and $a$ for RCP light in the whistler mode. The solid, dashed, dot-dashed and dotted curves show $B=1.1,1.5,2$ and 3 , respectively. The black points on each curve correspond to critical values $\left(a_{c}, \gamma_{c}\right)$ described in the text. Physically, $\gamma$ must always take the lowest value available when $a<a_{c}$. For $a>a_{c}$ the plasma is highly absorbing and the actual electron Lorentz factor can vary over many orders of magnitude.

\subsection{Transverse Properties}

Analytically we consider a regime where the laser pulse is relatively long so that the longitudinal motion of electrons due the longitudinal ponderomotive force can be neglected. Also we consider a regime where the laser intensity is weakly relativistic so that the density modification due to the transverse ponderomotive force can be neglected during the laser interaction. The numerical simulations detailed in section 3 show that this approximation is correct during the early stages of propagation, although nonlinear evolution at later stages leads to the development of very complicated structures that cannot be described by this analytical model.

Consider a generalised laser pulse with a form similar to (2);

$\boldsymbol{a}=\Re\{\hat{\boldsymbol{e}} a(r, z, t) \exp [i(k z-\omega t)]\}$,

where now the amplitude term is replaced by a slowly-varying envelope function $a(r, z, t)$. The evolution equation of the laser envelope is obtained by substituting (10) into (6), and evaluating it under the slowly varying envelope approximation (SVEA) [16]. Let $a(r, z, t)=a(r, \xi, \tau)$, where $\tau=t$ and $\xi=z-v_{g} t$ with the assumption that $v_{p} v_{g} \sim c^{2}$, and therefore $v_{g} \sim k c^{2} / \omega$. Then one can find

$\left(2 i \frac{\partial}{\partial \tau}+\nabla_{\perp}^{2}+\sigma \frac{\omega_{p}^{2}}{\omega^{2}} \frac{\partial^{2}}{\partial \xi^{2}}\right) a=\left(\frac{n_{e} \eta}{\gamma}-\sigma\right) a$, 

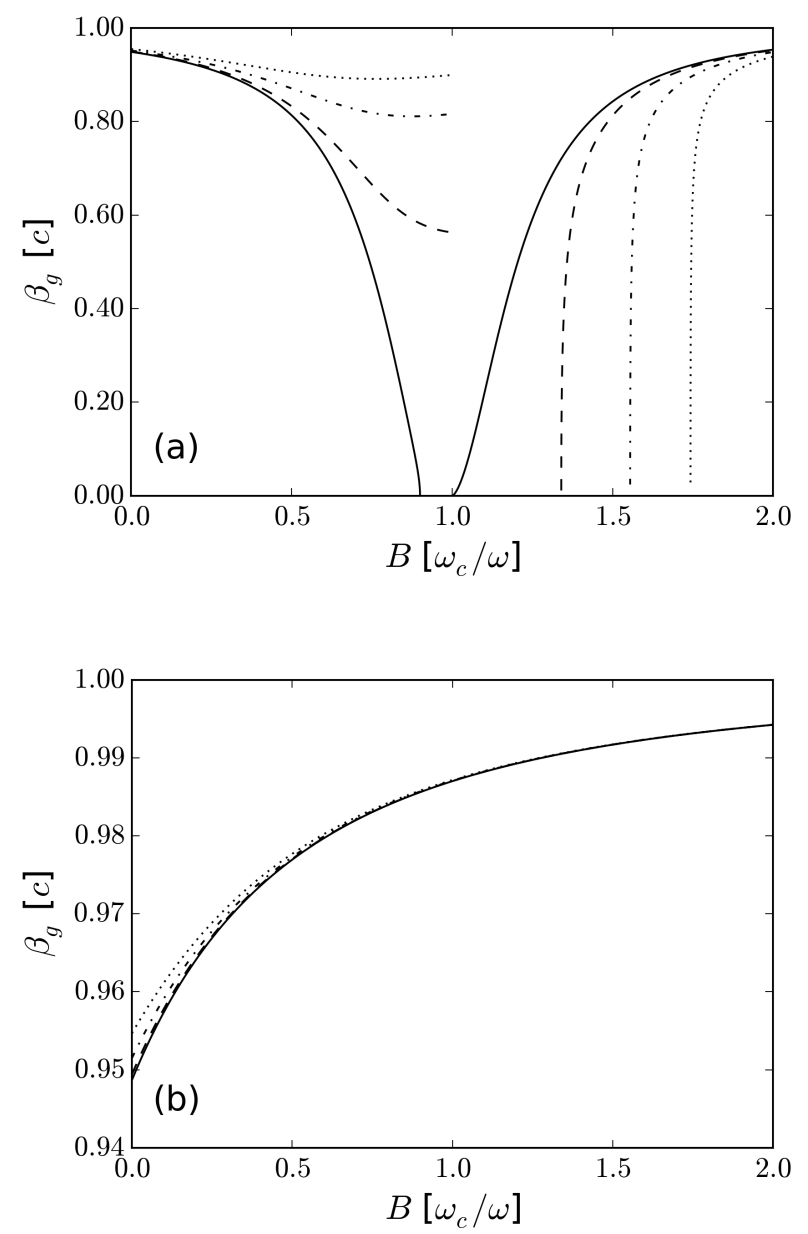

Figure 3: Calculated group velocity for light of varying intensity in a plasma of constant density $n=0.1$. the solid, dashed, dot-dashed and dotted lines correspond to $a=0,0.1,0.2$ and 0.3 , respectively. (a) RCP light. The values of $\beta_{g}$ when $B \simeq 1$ are in reality quite different from those calculated due to the finite nature of real pulses. Plasma absorption and heating begins before the peak of the pulse can act to reduce to effective cyclotron frequency. (b) LCP light. Tends asymptotically towards $\beta_{g}=1$ with increasing $B$.

where $\nabla_{\perp}^{2} a=r^{-1} \partial / \partial r(r \partial a / \partial r)$ is the radial component of the Laplacian and the normalisations $\tilde{\tau}=\left(\omega_{p}^{2} / \omega\right) \tau, \tilde{\xi}=k_{p} \xi$, and $\tilde{r}=k_{p} r$ have been used, with the tildes henceforth dropped for convenience. In the above equation, we discard second order derivatives $\partial^{2} / \partial \tau^{2}$ and $\partial^{2} / \partial \tau \partial \xi$ in accordance with the SVEA, and use a generic dispersion relation for a light beam with finite transverse width $\omega^{2}=k^{2} c^{2}+\sigma \omega_{p}^{2}$ with $\sigma$ a constant associated with the laser power [14]. In this equation, $\nabla_{\perp}^{2}$ represents the transverse diffraction effect and $\partial^{2} / \partial \vec{\xi}^{2}$ the longitudinal spreading due to linear plasma dispersion. The right hand side of this equation is a nonlinear term, leading to self-focusing and pulse compression.

To obtain the laser power threshold for selffocusing, one may consider a stationary solution and a laser pulse with long longitudinal profile. To further simplify calculations, an analytical form for the electron Lorentz factor is required. The weakly relativistic approximation assumes that $\gamma$ remains close to 1 . This is usually satisfied when $a^{2} \ll 1$. However, magnetisation introduces the additional factor $\eta$. If we consider $\gamma \sim 1$, then $\eta \sim \eta_{0}=(1-\delta B)^{-1}$. When $B \simeq 1, \eta_{0}^{2} \gg 1$ and so we must modify the conditions to $a^{2} \eta_{0}^{2} \ll 1$, under which we may consider the weaklyrelativistic approximation to be still satisfied. This effectively limits the scope of application of this theory to only the low- $B$ or very high- $B$ regime for RCP light, but increases the amplitude range we may consider for strongly magnetised LCP light.

Performing a Taylor expansion of (4) yields

$\gamma \approx 1+\frac{a^{2} \eta_{0}^{2}}{2}$

Substituting $\partial a / \partial \tau=0, \partial^{2} a / \partial \xi^{2}=0$ and (12) into (11) with the approximation $\eta / \gamma \approx \eta_{0}-a^{2} \eta_{0}^{4} / 2$, one can obtain the stationary envelope equation for a laser beam in a magnetised plasma

$\nabla_{\perp}^{2} a-\left(\eta_{0}-\sigma\right) a+n_{e} \frac{\eta_{0}^{4}}{2} a^{3}=0$,

At threshold, we assume the ponderomotive force to be small, and electron expulsion can be neglected, setting $n_{e}=1$. We may recast (13) via a change of variables, according to the general form $\nabla_{\perp}^{2} a-\epsilon_{1} a+\epsilon_{2} a^{3}=0$, where $\epsilon_{1}$ and $\epsilon_{2}$ are arbitrary coefficients. We may use the relations $a=\alpha\left(\epsilon_{1} / \epsilon_{2}\right)^{1 / 2}$ and $r=\rho \epsilon_{1}^{1 / 2}$ to transform between $a(r)$ and $\alpha(\rho)$, where $\alpha(\rho)$ is the solution of $a(r)$ with $\epsilon_{1}=\epsilon_{2}=1$. This may be evaluated numerically with the boundary conditions $\mathrm{d} \alpha(0) / \mathrm{d} \rho=0$ and $\alpha(\infty)=0$. Recasting and evaluating (13) for the beam power via the relation between amplitude and intensity in vacuum $I=\epsilon_{0} c|E|^{2}$, numerically evaluating and reverting to dimensional quantities yields the critical power for self-focusing

$P_{c}=1.62 \times 10^{10} \eta_{0}^{-4}\left(\frac{\omega}{\omega_{p}}\right)^{2}[W]$.

This result is consistent with previous work $[16,30]$ i.e. focusing effects are stronger at higher densities, and the additional term $\eta_{0}^{-4}$ relates to the magnetic field. This term reduces to unity for $B=0$, making the result generally applicable. The corrective term suggests that the magnetic field strongly alters the threshold for self-focusing by a factor of $\eta_{0}^{-4}=(1-\delta B)^{4}$. The form of the adjustment is reversed for RCP and LCP light and is shown in Figure 4. It shows that the $\mathrm{SF}$ power threshold is significantly reduced for RCP 


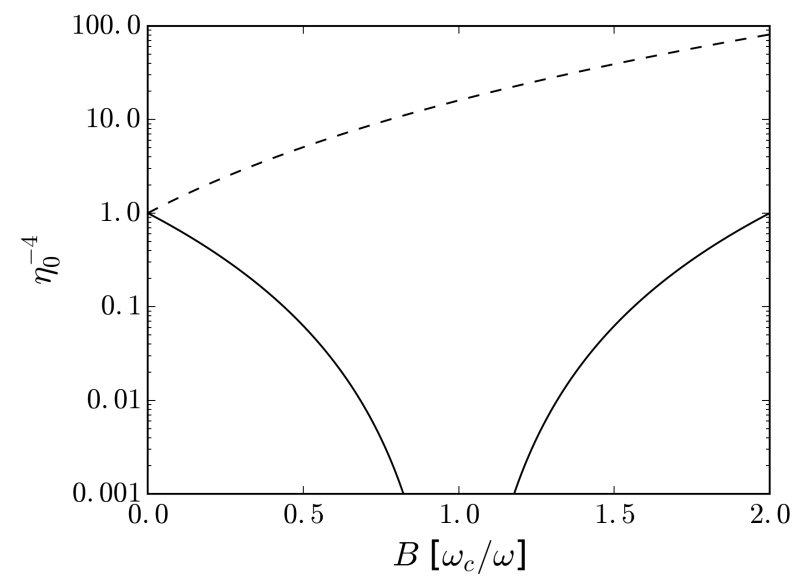

Figure 4: The adjustment to the critical power for selffocusing as induced by an applied magnetic field. The solid curve represents the adjustment for RCP light, the dashed curve for LCP light.

light near $B \sim 1$. This analysis implies that at $B=1$ the threshold power drops to nothing. In reality however, this is the point at which cyclotron resonance occurs, and the plasma becomes opaque in the lowpower regime and strongly absorbing at high light power. Resonant effects preclude the exploitation of this parameter regime for anything other than plasma heating. Conversely for LCP light, the SF power threshold increases monotonically with $B$.

Alternatively, starting with Eq. (11), assuming a Gaussian transverse amplitude profile such as by $a(\tau)=A(\tau) \exp \left(-r^{2} / R^{2}(\tau)\right)$, it can be shown, by either source-dependant expansion [32] or variational methods [33], that the beam waist evolution of a $\mathrm{CP}$ laser pulse can be described by

$\frac{\mathrm{d}^{2} R}{\mathrm{~d} \tau^{2}}=\frac{4}{k^{2} R^{3}}\left(1-\frac{P}{P_{c}}\right)$

where critical power is given by $P_{c}=1.74 \times$ $10^{10} \eta_{0}^{-4}\left(\omega / \omega_{p}\right)^{2}[\mathrm{~W}]$, which is only slightly different from that given in (14).

\subsection{Longitudinal Properties}

For short pulses, one can consider the evolution of the longitudinal profile of a laser pulse in magnetised plasmas due to SPM by use of (11). The phase change due to nonlinearity on the right hand side of this equation is given by

$\Delta \phi=-\frac{1}{2} \int^{\tau}\left(\frac{n_{e} \eta}{\gamma}-\sigma\right) d \tau^{\prime}$

The corresponding frequency change is given by $\Delta \omega=-\partial(\Delta \phi) / \partial t$. Alternatively, the effect can be demonstrated directly for a specific set of parameters.
For a temporal pulse profile $a(t)$, the instantaneous phase of the wave after propagating a distance $L$ into a homogeneous plasma is given by $\phi=\omega t-N k L$. In this case the frequency change may be estimated from $\omega=\partial \phi / \partial t$ as

$\Delta \omega=-\frac{\pi L}{N} \frac{n_{e}}{n_{c}}\left(\frac{a \eta^{4}}{\gamma^{3}+\delta B a^{2} \eta^{3}}\right) \frac{\partial a}{\partial t}$,

where $L$ is normalised to $2 \pi / k$. This is illustrated for RCP and LCP light in Figure 5. The SPM of the laser pulse leads to the compression of the longitudinal profile of the laser pulse. In the weakly relativistic case, the compression level is found by letting $\left(\omega_{p}^{2} / \omega^{2}\right)\left(\partial^{2} / \partial \xi^{2}\right) \sim \eta_{0}^{4} a^{2} / 2$ in (11). Therefore, the laser pulse duration can be compressed to the level

$L_{p} \approx \frac{\sqrt{2}}{k_{p}} \frac{\omega_{p}}{\omega}\left(\eta_{0}^{2} a\right)^{-1}$.

This suggests that the longitudinal compression can be significantly enhanced for RCP light with $B<1$. The magnitude of SPM is tied heavily to the plasma density, with higher density producing stronger modulation.

Pulse compression by SPM alone is not possible for whistler-mode lasers. If we recall that $B=\omega_{c} / \omega$, and take $\omega_{c}$ to be constant, $B$ will vary inversely with $\omega$. By analysis of (8) (or inspection of Figure 3a), we can find that $\partial \beta_{g} / \partial \omega$ takes opposite signs for $B<1$ and $B>1$. When this is considered alongside the frequency shift given by (17), it can hence be shown that the effect of SPM is reversed when $B>1$, acting to lengthen the pulse, rather than compress it.

\section{Numerical simulation results}

To illustrate the key features of laser pulse propagation in strongly magnetised plasma predicted by theory discussed above, we have carried out $1 \mathrm{D}$ and $3 \mathrm{D}$ particle-in-cell (PIC) simulations. All of the following numerical simulations are performed using the PIC code OSIRIS. In general length scales are characterised by the laser wavelength $\lambda_{0}$ in vacuum and timescales by the laser period $\tau_{0}=\lambda_{0} / c$.

For 3D simulations, we use a Cartesian coordinate system, with a grid resolution set to 20 cells per wavelength in the longitudinal direction, and 5 cells per wavelength in the transverse directions. In order to simulate long propagation distances it is convenient to make use of the moving window feature of OSIRIS. The laser pulse propagates in the forward $x$ direction. It has a pure sine-squared longitudinal profile with the initial pulse duration of $50 \tau_{0}$ in full-width. The laser is initialised in vacuum and modelled as a Gaussian beam with a waist size at the focal plane of $r_{0}=10 \lambda_{0}$. The plasma is initially homogeneous after a short ramp of $1 \lambda_{0}$ to minimise boundary reflection and the focal plane is located at the start of the plasma density plateau. 

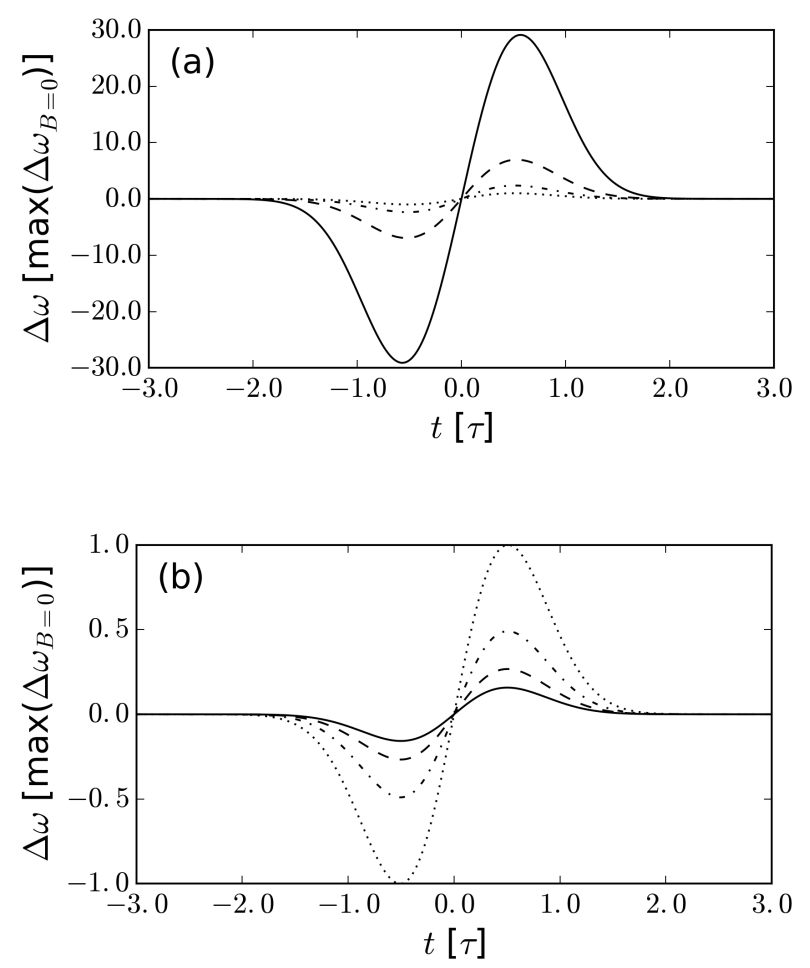

Figure 5: Frequency shift introduced by SPM for a Gaussian envelope $a=a_{0} \exp \left(-t^{2} / \tau^{2}\right)$. We show a pulse of amplitude of $a_{0}=0.1$, at an arbitrary distance $L$ into a plasma of density $n=0.1$. The dotted line plot shows the unmagnetised case. The influence of an external magnetic field causes an increase in frequency spread for the same amplitude and density. The dotdashed, dashed and solid lines correspond to $B=0.2$, 0.4 and 0.6 , respectively. The y-axis is scaled to the unmagnetised case. (a) RCP. (b) LCP.

There are 4 initially cold macroparticles per cell while ions are assumed to be immobile, accounting for the fact that the laser pulse duration is short. In 1D, the resolution is increased to 400 cells per wavelength, and 25 particles per cell. Where possible, all other parameters match the $3 \mathrm{D}$ simulations.

In terms of normalised peak amplitude, the power of circularly polarised light with a Gaussian transverse profile in the lowest mode is given by $P=4.29 \times 10^{10}\left(a_{0} r_{0} / \lambda_{0}\right)^{2}[\mathrm{~W}]$. The conditions for selffocusing are met when $P / P_{c}>1$ where $P_{c}$ is given by (14).

To test the effect of a magnetic field on the evolution of a RCP laser, we consider a plasma with plateau density $\left(\omega_{p} / \omega\right)^{2}=0.02$, with an external magnetic field of $B=0.5$ (approximately $5 \times 10^{3}$ Tesla for $\left.\lambda_{0}=1 \mu \mathrm{m}\right)$. The predicted self-focusing adjustment is $\eta_{0}^{-4}=1 / 16$ that of the unmagnetised case, corresponding to a peak irradiance of approximately
$3 \times 10^{16} \mathrm{Wcm}^{-2}$ (for $\left.\lambda_{0}=1 \mu \mathrm{m}\right)$. We use a laser with initial peak amplitude of $a_{0}=0.15$, such that $P / P_{c} \approx 2$. The results of this simulation are shown in figure 6 . The pulse propagates at reduced $\beta_{g}$ according to Eq. (8). Both SF and SPM-driven longitudinal compression are visible, which are coupled to each other. These effects cause a large increase in peak intensity, close to 6 times the initial amplitude. This increase in amplitude causes the excitation of longitudinal electron plasma waves at the wavelength of $2 \pi v_{g} / \omega_{p}$, which has been reduced as compared to unmagnetised plasma according to figure 3(a). The plasma waves further distort the longitudinal profile of the laser pulse, resulting in the formation of a pulse train similar to that studied by $[34,35]$. As the beam waist collapses down further, ponderomotive expulsion becomes significant, with the minimum electron density reaching close to, but not quite zero at the pulse. As expected, the self-generated magnetic field remains comparatively small, reaching only $2 \%$ of the imposed longitudinal magnetic field strength.

Figures 6(b-d) show that the pulse evolution initially appears to conform to the analytical model derived here. However, after a certain distance the pulse shape is radically altered, complex structures form and plasma heating begins. From these simulations it is evident that RCP pulse evolution in near resonant plasma is not well described by the model employed here. At even later time, longitudinal compression of the laser pulse occurs, which allows for the excitation of electron plasma waves [36], an effect which is explicitly ignored in deriving (4). The strong spatiotemporal compression also raises the pulse amplitude well beyond the weakly-relativistic regime. Obviously this continuous evolution of RCP pulses in the parameter regime $B \sim 1$ and $P>P_{c}$ is much beyond our analytical model. The model remains, however, a reasonable method to predict the initial tendencies of the pulse evolution.

An equivalent simulation was performed for LCP light. Laser pulse, plasma and magnetic field parameters remain the same with the exception that the beam irradiance is increased to $5 \times 10^{18} \mathrm{Wcm}^{-2}$, to maintain $P / P_{c} \approx 2$. The results of this simulation are shown in Figure 7. Focusing and compressional effects are smooth and the plasma electron response remains adiabatic throughout. The pulse group velocity is seen to be increased, in agreement with Eq. (8) and Figure 3(a). This can be qualatatively confirmed by inspection of Figures 6(d) and 7(d). Comparing the propagation distances of both pulses after the same amount of time highlights the difference in group velocity for LCP and RCP light.

It is well documented that plasma electrons gain significant amounts of energy near cyclotron resonance, 
(a)

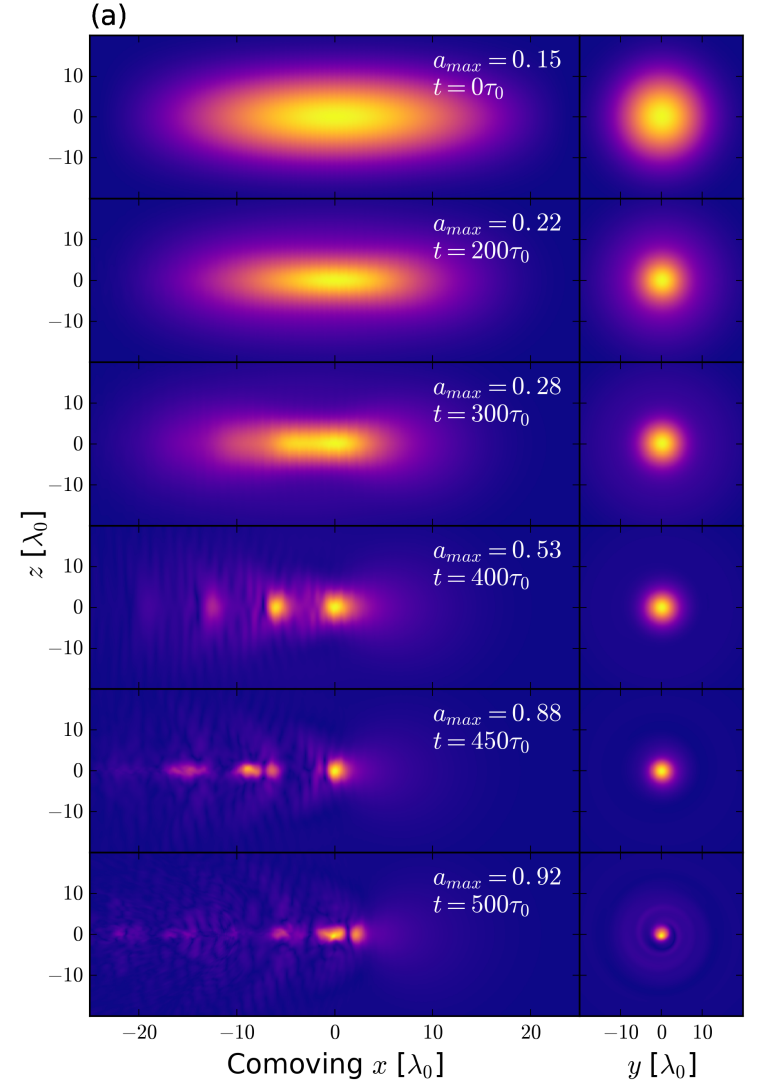

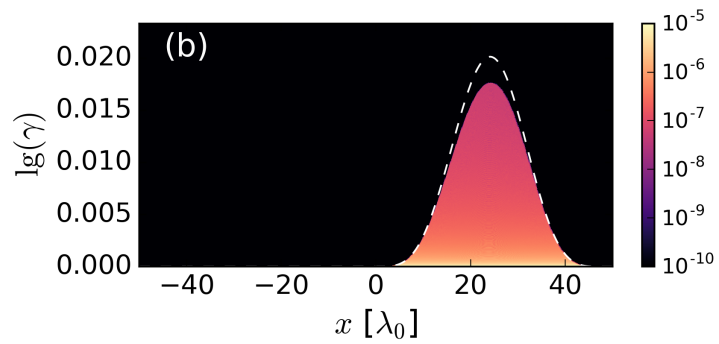
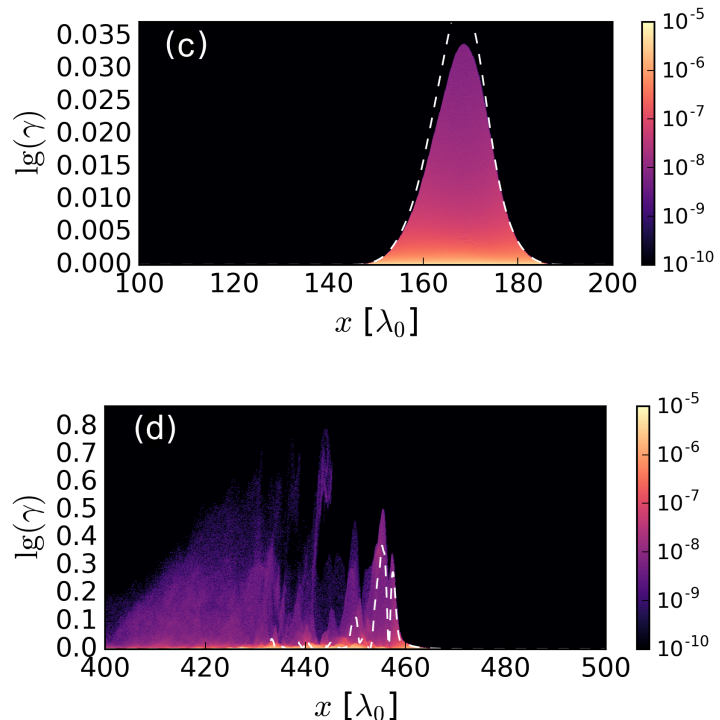

Figure 6: Simulation results for a RCP pulse with $B=0.5, P / P_{c} \approx 2$. (a) Pulse evolution over time. The left column shows the longitudinal amplitude profile, the right column shows the transverse amplitude profile at the longitudinal peak amplitude. Each row is independently normalised, with the colour scale ranging from 0 to the peak amplitude indicated by the value $a_{\max }$ on each row. $(\mathrm{b}-\mathrm{d})$ The normalised electron distribution at $t=$ $50 \tau_{0}, 200 \tau_{0}$ and $500 \tau_{0}$, respectively. The dashed line plots denote the the prediction by (12) using the on-axis pulse amplitude. Focusing and compression are observed over the first few hundred laser periods, until density modulations cause pulse breakup into a train of very short, highly amplified pulses of the plasma wavelength in length. The pulse train undergoes catastrophic collapse and loses cohesion, diffusing rapidly after this. The beginning of this process can be seen at $t=500 \tau_{0}$. This also marks the point at which the electron response ceases to be adiabatic.

which finds application in resonance heating systems for magnetically confined fusion reactors [37]. Here we are relatively far from resonance and still observe large amounts of energy absorption to the plasma, with the peak electron Lorentz factor observed to be around 3 times that predicted by the theory. This behaviour is more problematic in the near-resonant whistler regime, as intense light may easily cause a feedback of electrons continually gaining energy, resulting in the plasma returning to a resonant state. The asymmetry in electron response around $B=1$ is not predicted by the simple model in (12) but may be readily observed in simulation.

To demonstrate the critical amplitude as described by (9) we performed a $1 \mathrm{D}$ simulation of a whistler pulse, shown in Figure 8. For $B=2, a_{c}=0.45$ and hence the initial peak amplitude was set at $a_{0}=0.5$. The pulse was also lengthened to $100 \tau_{0}$ in full-width so as to better demonstrate the process.

As the pulse moves through the plasma, the leading edge produces an adiabatic electron response in agreement with the theory, up until the pulse amplitude $a=a_{c}$. At this point, the plasma becomes highly absorbing and the rear of the pulse is rapidly absorbed. This leaves an attenuated pulse traveling with a wake of heated plasma behind it. While this is very well represented in $1 \mathrm{D}$, extension to higher dimension introduce transverse effects which alter the 
(a)

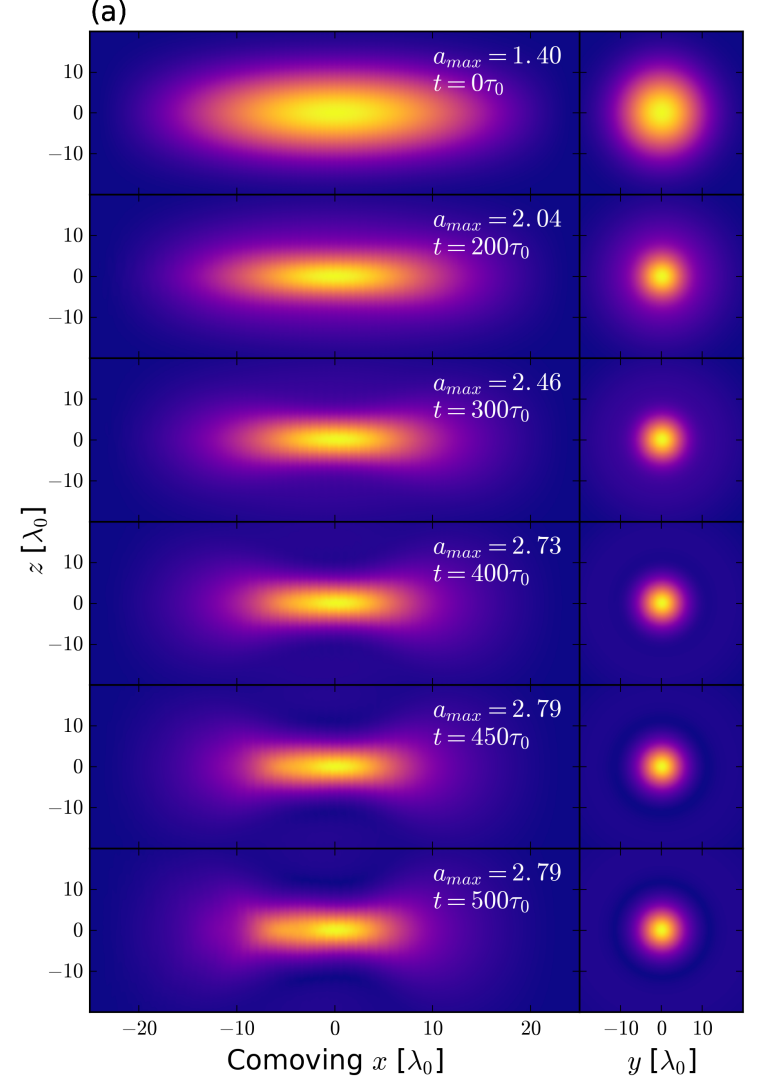

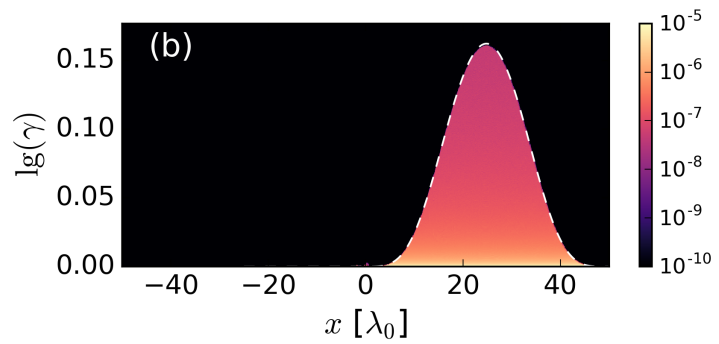
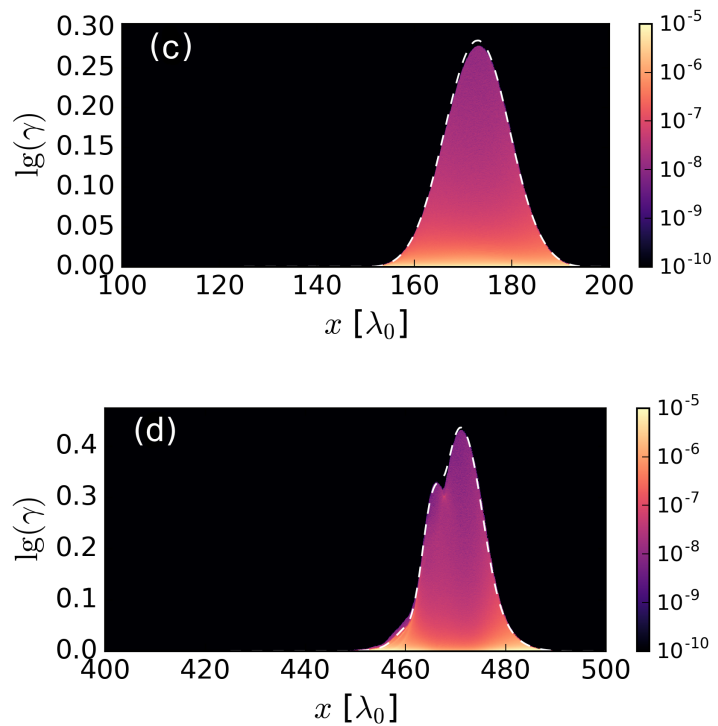

Figure 7: Simulation results for a LCP pulse with $B=0.5, P / P_{c} \approx 2$. (a) Pulse evolution over time. The left column shows the longitudinal amplitude profile, the right column shows the transverse amplitude profile at the longitudinal peak amplitude. Each row is independently normalised, with the colour scale ranging from 0 to the peak amplitude indicated by the value $a_{\max }$ on each row. $(\mathrm{b}-\mathrm{d})$ The normalised electron distribution at $t=$ $50 \tau_{0}, 200 \tau_{0}$ and $500 \tau_{0}$ respectively. The dashed line plots denote the the prediction by (12) using the on-axis pulse amplitude. Smooth focusing and compression occur down to an equilibrium spot size. In the later stages, this pulse begins to be modulated by density fluctiations in a similar manner to the RCP pulse (Figure 6).

propagation characteristics. However, the critical amplitude remains a good estimate of the onset of energy absorption to the plasma.

\section{Conclusions}

The theory and simulation results presented here have demonstrated the influence an externally applied magnetic field has on weakly relativistic plasma electron dynamics and laser propagation. In general, LCP light experiences dampened electron motion, and hence a reduction in instability growth in the presence of a strong external magnetic field. Under these conditions, pulse evolution becomes less sensitive to pulse amplitude, and the weakly relativistic approximation remains applicable even at high pulse amplitudes.
In contrast to this, RCP light will experience dramatically enhanced electron motion which becomes unpredictable when the electron cyclotron frequency approaches the incident laser frequency $B / \gamma \simeq 1$. As $B$ enters the whistler regime electron motion is once more reduced, and exceptionally strong fields of $B>2$ experience reduced electron motion compared to the unmagnetised case for both polarisation directions.

Near to cyclotron resonance, electron motion and hence pulse evolution is very sensitive to laser amplitude for RCP light. This puts considerable strain on remaining under the weakly-relativistic approximation. Even a pulse which is initially weakly relativistic can quickly evolve to a point at which it can no longer be considered as such. This introduces complicated nonlinear phenomena, in particular, strong coupling between transverse and 


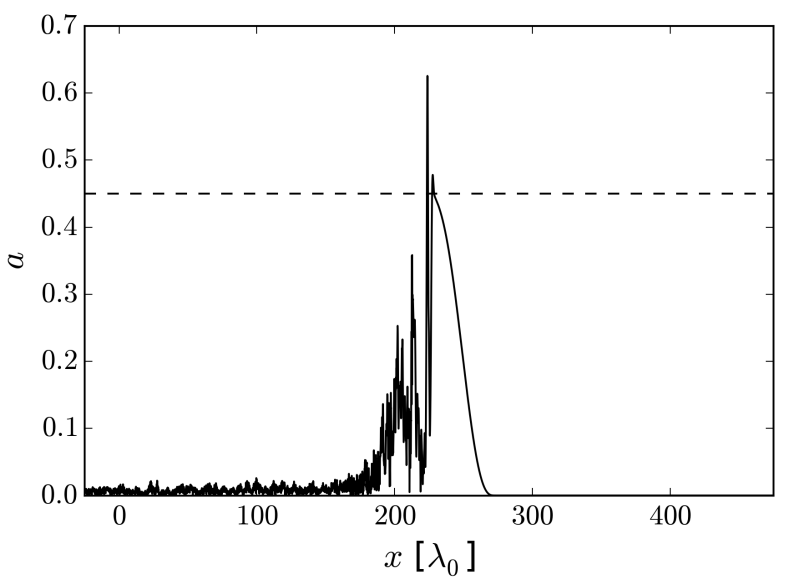

Figure 8: A pulse of initial amplitude $a_{0}=0.5$ and length $100 \tau_{0}$ after propagating around $200 \lambda_{0}$ into a plasma with $B=2$. The dashed line marks the critical amplitude $a_{c}$ as given by (9). The rear of the pulse is seen to be experiencing absorption by the plasma, while the leading edge is propagating without any absorption.

longitudinal evolution in the short pulse regime. Transverse focus increases the peak amplitude of the pulse, which in turn enhances longitudinal pulse compression, ponderomotive expulsion of electrons, excitation of electron plasma waves and subsequent electron acceleration. These are beyond the scope of the presented analytical model.

Despite this, the combination of transverse and longitudinal effects on RCP laser pulses may offer new opportunities for the manipulation of high power laser propagation in underdense plasmas, which may be relevant to a few applications such as plasma heating for fusion, laser-plasma based particle acceleration and radiation.

\section{Acknowledgments}

The authors acknowledge the OSIRIS consortium, a Leverhulme Trust Research Project Grant, the U.K. EPSRC (Grant No. EP/N028694/1), and EuPRAXIA (Grant No. 653782). Numerical simulations have been performed in ARCHER via Plasma HEC Consortium supported by EPSRC (No. EP/L000237/1).

\section{References}

[1] X. H. Yang, W. Yu, H. Xu, M. Y. Yu, Z. Y. Ge, B. B. Xu, H. B. Zhuo, Y. Y. Ma, F. Q. Shao, and M. Borghesi. Propagation of intense laser pulses in strongly magnetized plasmas. Applied Physics Letters, 106:224103, 2015.
[2] K. Mima et al. Magnetized fast ignition (mfi) and laser plasma interaction in strong magnetic field. Journal of Physics: Conference Series, 688:012066, 2013.

[3] F. Debray and P. Frings. State of the art and developments of high field magnets at the 'laboratoire national des champs magntiques intenses'. Comptes Rendus Physique, 14:2, 2013.

[4] J R Sims, D G Rickel, C A Swenson, J B Schillig, and C N Ammerman. Assembly, commissioning and operation of the nhmfl 100 tesla multi-pulse magnet system. IEEE Transactions on Applied Superconductivity, 18:587, 2008.

[5] P. F. Schmit et al. Understanding fuel magnetization and mix using secondary nuclear reactions in magnetoinertial fusion. Physical Review Letters, 113:155004, 2014.

[6] U. Wanger et al. Laboratory measurements of $0.7 \mathrm{gg}$ magnetic fields generated during high-intensity laser interactions with dense plasmas. Physics of Plasmas, 70:026401, 2004.

[7] A S Sandhu et al. Direct observation of turbulent magnetic fields in hot, dense laser produced plasmas. Phys. Rev. Lett., 89:225002, 2002.

[8] S Mondal et al. Direct observation of turbulent magnetic fields in hot, dense laser produced plasmas. Proc. Natl Acad. Sci., 109:8011, 2012.

[9] A D Shaikh et al. Megagauss magnetic fields in ultraintense laser generated dense plasmas. Plasma Phys. Control. Fusion, 59:014007, 2017.

[10] S Fujioka et al. Kilotesla magnetic field due to a capacitorcoil target driven by high power laser. Scientific Reports, 3:1170, 2013.

[11] J. J. Santos et al. Laser-driven platform for generation and characterization of strong quasi-static magnetic fields. New Journal of Physics, 17:083051, 2015.

[12] W. M. Wang, P. Gibbon, Z. M. Sheng, and Y. T. Li. Magnetically assisted fast ignition. Phys. Rev. Lett., 114:015001, 2015.

[13] R. A. Helliwel. Whistlers and Related Ionospheric Phenomena. Stanford University Press, 2014.

[14] G.-Z. Sun, E. Ott, Y. C. Lee, and P. Guzdar. Self-focusing of short intense pulses in plasmas. Physics of Fluids, 30:526, 1987.

[15] P. Sprangle, E. Esarey, A. Ting, and G. Joyce. Laser wakefield acceleration and relativistic optical guiding. Applied Physics Letters, 53:2146, 1988.

[16] A. B. Borisov, A. V. Borovskiy, O. B. Shiyaev, V. V. Korobkin, A. M Prokhorov, J. C. Solem, T. S. Luk, K. Boyer, and C. K. Rhodes. Relativistic and chargedisplacement self-channeling of intense ultrashort laser pulses in plasmas. Physical Review A, 45:5830, 1992.

[17] O. Shorokhov and A. Pukhov. Self-compression of laser pulses in plasma. Physical Review Letters, 91:265002, 2003.

[18] I. Watts, M. Zepf, E. L. Clark, M. Tatarakis, K. Krushelnick, and A. E. Dangor. Measurements of relativistic self-phase modulation in plasma. Physical Review E, 66:036409, 2002

[19] M. Malekshahi, D. Dorranian, and H. R. Askari. Selffocusing of the high intensity ultra short laser pulse propagating through relativistic magnetized plasma. Optics Communications, 332:227, 2014.

[20] A. Sharma and V. K. Tripathi. Relativistic and ponderomotive self-focusing of a laser pulse in magnetized plasma. Laser and Particle Beams, 30:659, 2012.

[21] Y. Liang, H.-B. Sang, F. Wan, C. Lv, and B.-S. Xie. Relativistic laser pulse compression in magnetized plasmas. Physics of Plasmas, 22:073105, 2015.

[22] B. Bokaei, A. R. Niknam, and E. Imani. Spatiotemporal evolution of high power laser pulses in relativistic mag- 
netized inhomogeneous plasmas. Physics of Plasmas, 22:092310, 2015.

[23] T. S. Gill, R. Kaur, and R. Mahajan. Self-focusing of super-gaussian laser beam in magnetized plasma under relativistic and ponderomotive regime. Optik, 126:1683, 2015.

[24] L Gorbunov, P Mora, and T M Antonsen. Magnetic field of a plasma wave driven by a laser pulse. Physical Review Letters, 76:2495, 1996.

[25] A Pukhov and J Meyer-ter Vehn. Relativistic magnetic self-channeling of light in near-critical plasma: Threedimensional particle-in-cell simulation. Physical Review Letters, 76:3975, 1996.

[26] Z M Sheng and J Meyer-ter Vehn. Analytic and numerical study of magnetic fields in the plasma wake of an intense laser pulse. Physics of Plasmas, 5:3764, 1998.

[27] V I Berezhiani, S M Mahajan, and N L Shatashvili. Theory of magnetic field generation by relativistically strong laser radiation. Physical Review E, 55:995, 1997.

[28] I Kostyukov, G Shvets, N J Fisch, and J M Rax. Inverse faraday effect in a relativistic laser channel. Laser and Particle Beams, 19:133, 2001.

[29] J A Stamper and B H Ripin. Faraday-rotation measurements of megagauss magnetic fields in laser-produced plasmas. Physical Review Letters, 34:138, 1975.

[30] Z.-M. Sheng and J. Meyer-ter Vehn. Inverse faraday effect and propagation of circularly polarized intense laser beams in plasmas. Physical Review E, 54:1833, 1996.

[31] S. X. Luan, W. Yu, F. Y. Li, D. Wu, Z. M. Sheng, M. Y. Yu, and J. Zhang. Laser propagation in dense magnetized plasma. Phys. Rev. E, 94:053207, 2016.

[32] P. Sprangle, A. Ting, and C. M. Tang. Analysis of radiation focusing and steering in the free-electron laser by use of a source-dependent expansion technique. Physical Review A, 36:2773, 1987.

[33] D. Anderson and M. Bonnedal. Variational approach to nonlinear self-focusing of gaussian laser beams. Physics of Fluids, 22:105, 1979.

[34] E Esaray, J Krall, and P Sprangle. Envelope analysis of intense laser pulse self-modulation in plasmas. Phys. Rev. Lett., 72:2887, 1994.

[35] Z.-M. Sheng, K. Mima, Y. Sentoku, Nishihara K., and J. Zhang. Generation of high amplitude plasma waves for particle acceleration by cross-modulated laser wake fields. Physics of Plasmas, 9:3147, 2002.

[36] P Mora and T M Antonsen. Electron cavitation and acceleration in the wake of an ultraintense, self-focused laser pusle. Pyhsical Review E, 53:R2068, 1996.

[37] V. Erckmann and U. Gasparino. Electron cyclotron resonance heating and current drive in toroidal fusion plasmas. Plasma Physics and Controlled Fusion, 36:1869, 1994. 